\title{
Characterisation of GLUT4 trafficking in HeLa cells: comparable kinetics and orthologous trafficking mechanisms to 3T3-L1 adipocytes
}

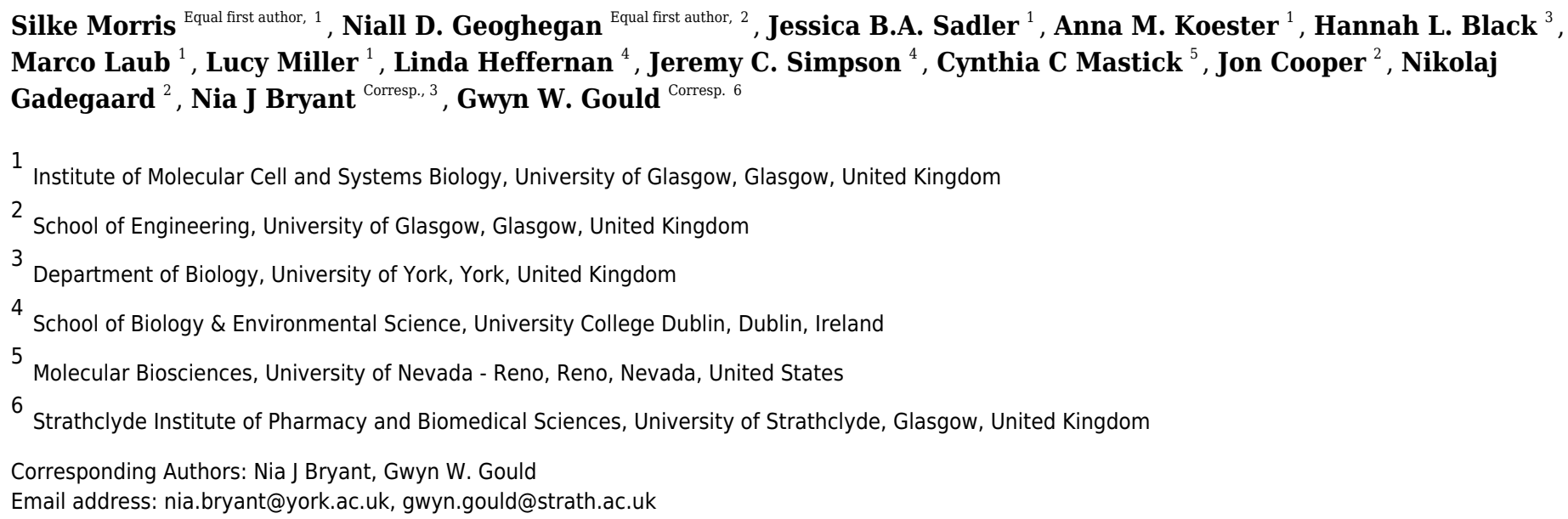

Insulin-stimulated glucose transport is a characteristic property of adipocytes and muscle cells and involves the regulated delivery of glucose transporter (GLUT4)-containing vesicles from intracellular stores to the cell surface. Fusion of these vesicles results in increased numbers of GLUT4 molecules at the cell surface. In an attempt to overcome some of the limitations associated with both primary and cultured adipocytes, we expressed an epitope- and GFP-tagged version of GLUT4 (HA-GLUT4-GFP) in HeLa cells. Here we report the characterisation of this system compared to 3T3-L1 adipocytes. We show that insulin promotes translocation of HA-GLUT4-GFP to the surface of both cell types with similar kinetics using orthologous trafficking machinery. The utility of the HeLa cell system is exemplified by a small-scale siRNA screen which identifies GOSR1 and YKT6 as potential novel regulators of GLUT4 trafficking in human cells. 
Characterisation of GLUT4 trafficking in HeLa cells: comparable kinetics and orthologous trafficking mechanisms to 3T3-L1 adipocytes.

3

4

Silke Morris ${ }^{1}$, Niall D. Geoghegan ${ }^{2}$, Jessica B.A. Sadler ${ }^{1}$, Anna M. Koester ${ }^{1}$, Hannah L. Black $^{3}$, Marco Laub ${ }^{1}$, Lucy Miller ${ }^{1}$, Linda F. Heffernan ${ }^{4}$, Jeremy C. Simpson ${ }^{4}$, Cynthia C. Mastick $^{1,5}$, Jon Cooper ${ }^{2}$, Nikolaj Gadegaard ${ }^{2}$, Nia J. Bryant ${ }^{3, *}$ and Gwyn W. Gould ${ }^{1,6, *}$.

${ }^{1}$ Henry Welcome Laboratory for Cell Biology, Institute for Molecular, Cellular and Systems Biology, College of Medical Veterinary and Life Sciences, University of Glasgow. United Kingdom.

${ }^{2}$ School of Engineering, Rankine Building, College of Science and Engineering, University of Glasgow. United Kingdom.

${ }^{3}$ Department of Biology, University of York. United Kingdom.

${ }^{4}$ School of Biology \& Environmental Science, University College Dublin, Ireland.

${ }^{5}$ Molecular Biosciences, University of Nevada Reno, USA.

*to whom correspondence should be addressed. gwyn.gould@,strath.ac.uk or nia.bryant@york.ac.uk

${ }^{6}$ present address: Strathclyde Institute for Pharmacy and Biomedical Sciences, University of Strathclyde, Glasgow. United Kingdom.

\section{Abstract}

Insulin-stimulated glucose transport is a characteristic property of adipocytes and muscle cells and involves the regulated delivery of glucose transporter (GLUT4)-containing vesicles from intracellular stores to the cell surface. Fusion of these vesicles results in increased numbers of GLUT4 molecules at the cell surface. In an attempt to overcome some of the limitations associated with both primary and cultured adipocytes, we expressed an epitope- and GFP-tagged version of GLUT4 (HA-GLUT4-GFP) in HeLa cells. Here we report the characterisation of this system compared to 3T3-L1 adipocytes. We show that insulin promotes translocation of HAGLUT4-GFP to the surface of both cell types with similar kinetics using orthologous trafficking machinery. The utility of the HeLa cell system is exemplified by a small-scale siRNA screen which identifies GOSR1 and YKT6 as potential novel regulators of GLUT4 trafficking in human cells. 


\section{Introduction.}

Insulin-stimulated glucose transport in peripheral tissues is largely achieved by the delivery of intracellular glucose transport-4 (GLUT4)-containing vesicles to the cell surface where they dock and fuse (Bryant, Govers \& James, 2002; Jaldin-Fincati et al., 2017; Klip, McGraw \& James, 2019). This increases the numbers of functional transporters at the cell surface thereby raising the $\mathrm{V}_{\max }$ for glucose entry into the cell (Klip, McGraw \& James, 2019). Insulinstimulated glucose transport is impaired in Type-2 diabetes, providing a significant impetus into understanding the molecular mechanisms involved in this event (Kahn, 1992; Garvey et al., 1993 1998; Klip, McGraw \& James, 2019).

Many of the studies which investigate the mechanism of GLUT4 translocation use adipocytes either from animal or human tissues, or the 3T3-L1 adipocyte cell model. Primary tissue suffers from difficulty in employing many routine molecular manipulations (e.g. RNAi, over-expression etc.) as cells de-differentiate in culture over time, and the 3T3-L1 adipocyte cell line is difficult to transfect, requiring usually either electroporation or viral nucleic acid delivery mechanisms (Orlicky \& Schaack, 2001; Puri et al., 2007). Furthermore, 3T3-L1 adipocytes require extensive periods in culture which can make high throughput screening difficult.

Recently, we and others have established HeLa cells expressing epitope-tagged GLUT4 as a model system which facilitate studies into aspects of GLUT4 biology by virtue of the ease of genetic manipulation of this commonly employed cell line (Kawase et al., 2006; Haga, Ishii \& Suzuki, 2011; Kioumourtzoglou et al., 2015; Gulbranson et al., 2017; Camus et al., 2020). Here, we describe characterisation of these cells compared to 3T3-L1 adipocytes expressing the same GLUT4 reporter. We show that these two lines, while differing in the magnitude of the insulin response, show many similar characteristics. While we advocate working on cell types as close to physiologically relevant tissues as possible, we nevertheless believe our beta-testing analysis of HeLa cells expressing GLUT4 indicate these cells provide a useful system for initial trials or larger scale screening analyses which cannot be readily undertaken in either primary cells or differentiated cell culture systems. In support of this, we present data analysing the effects of vSNARE knockdown on GLUT4 trafficking and a small-scale RNAi screen for novel effectors of GLUT4 trafficking that suggest an important role for GOSR1 and YKT6 on GLUT4 trafficking and stability. We conclude that HeLa cells are a useful model for preliminary or high-throughput studies of GLUT4 traffic.

\section{Materials and Methods.}

Cells - HeLa and 3T3-L1 cells were obtained from the ATCC. A HeLa cell line stably expressing HA-GLUT4-GFP was created following infection with a lentiviral construct encoding GFP-tagged GLUT4 carrying an HA epitope in the first extracellular loop; clones were isolated by limited dilution (Muretta, Romenskaia \& Mastick, 2008; Muretta \& Mastick, 2009). 3T3-L1 adipocytes expressing the same construct were generated from 3T3-L1 fibroblasts engineered to express this construct stably as outlined (Muretta et al., 2008). Cells were grown and differentiated as previously described (Muretta, Romenskaia \& Mastick, 2008; Sadler, Bryant \& Gould, 2015). Cells were plated on a Total Internal Reflection Microscopy (TIRFM)compatible observation chambers (Ibidi, Glasgow) which contained a $170 \mu \mathrm{m}$ coverslip base. HeLa cells were plated to a density of $4 \times 10^{3}$ cells per chamber to distinguish single cells from the population for observation. Cells were left to attach to the surface of the coverslip overnight. Prior to measurement, all cells were serum starved for two hours. 
86

87

88

89

90

91

92

93

94

95

96

97

98

99

100

101

102

103

104

105

106

107

108

109

110

111

112

113

114

115

116

117

118

119

120

121

122

123

124

125

126

127

128

129

130

TIRFM - TIRFM images were acquired using an in-house constructed objective based TIRFM system. The light from a $481 \mathrm{~nm}$ diode laser (HORIBA) was directed to the far aperture of a 1.45 NA objective (Zeiss) using a Till Photonics TIRF condenser. The condenser contains a micrometre screw gauge for lateral manipulation of the beam relative to the optical axis. The resultant fluorescence light was collected by the same objective and focussed to an Andor Ixon EMCCD using a C-mount 1.6x expansion lens. The penetration depth of the evanescent field was measured using $10 \mu \mathrm{m}$ fluorescent particles and was found to be $110 \mathrm{~nm}$. For discrete membrane intensity imaging a series of 10 individual frames were acquired at each time point with an exposure time of $500 \mathrm{~ms}$. For all time-lapse image sets the rate of acquisition was set to 2 frames per second.

Live cell imaging - All cells were imaged in Ibidi chambers as above. A temperature of $37^{\circ} \mathrm{C}$ was maintained using a temperature control microscope insert (PeCon, Germany). Insulin stimulation was achieved by replacing $50 \%$ of the chamber's media with media containing 200 $\mathrm{nM}$ insulin to provide an overall concentration of $100 \mathrm{nM}$.

Membrane Associated Fluorescence Image Analysis - All image analysis was performed using the ImageJ/Fiji platform. For imaging of membrane associated fluorescence intensity, a perimeter was defined around the footprint of an individual cell for the initial images prior to insulin stimulation. Within this footprint the average pixel-wide fluorescence intensity was measured at discrete time points before and after the addition of $100 \mathrm{nM}$ insulin.

Mobile and static vesicle analysis - Images were processed as follows: The signal from GLUT4 vesicles versus the uneven diffuse fluorescent background was enhanced through the implementation of a rolling ball algorithm (Sternberg, 1983). Subsequently, noise was removed through the use of the 'de-speckle' and 'outlier removal' subroutines of ImageJ. To differentiate between static and mobile vesicles located at the membrane, image stacks were accumulated for 2 min. prior to insulin stimulation and for $25 \mathrm{~min}$. after. Stacks for individual cells were segmented to one-minute time bins for analysis. The average projection image was generated for each one-minute stack and was subtracted pixel by pixel from each image of the original stack. The resultant image stack contained data relating only to moving GLUT4-containing vesicles. This secondary image stack was subsequently subtracted from the original stack to provide a series of images containing only stationary vesicles.

Individual vesicles were defined by the following criteria using the FindFoci algorithm (i) that the fluorescent point had a local maxima value, (ii) $75 \%$ of the peak intensity was contained within a 5 pixel radius and (iii) the point was larger than a minimum 2 pixel radius (Herbert, Carr \& Hoffmann, 2014).

siRNA transfection - The day prior to transfection, cells were plated onto glass coverslips in a 24-well plate at a density of 7,500 cells/well. The following day, cells were transfected with 200 nM SMARTpool siRNAs (GE Healthcare Ltd.) specific for VAMP isoforms or Syntaxin-16, as indicated in the figure legends using DharmaFECT (ThermoFischer, UK), according to the manufacturer's instructions (Simpson et al., 2007; Simpson, 2009). Cells were assayed between 48-72h after transfection. Prior to use, cells were incubated in serum-free media for $2 \mathrm{~h} ; 1 \mu \mathrm{M}$ insulin was added for a further $20 \mathrm{~min}$. as indicated on the figure legends.

For cell lysates, transfected cells were washed twice with ice-cold PBS then solubilised in RIPA buffer: $20 \mathrm{mM}$ Tris- $\mathrm{HCl}$ (pH 7.5), $150 \mathrm{mM} \mathrm{NaCl}, 1 \mathrm{mM} \mathrm{Na}_{2}$ EDTA, $1 \mathrm{mM} \mathrm{EGTA}, 1 \%$ (v/v) NP$40,1 \%(\mathrm{v} / \mathrm{v})$ sodium deoxycholate, $2.5 \mathrm{mM}$ sodium pyrophosphate, $1 \mathrm{mM} \beta$-glycerophosphate, 1 $\mathrm{mM} \mathrm{Na} \mathrm{VO}_{4}$ and proteinase inhibitors. Anti-VAMP2 (\#104 202), anti-VAMP3 (\#104 103), anti- 
131 VAMP4 (\#136 002), anti-VAMP5 (\#176 003), anti-VAMP7 (\#232 003) and anti-VAMP8 (\#104

132 302) were from Synaptic Systems, Germany; gels and blots were performed as in (Sadler, Bryant

$133 \&$ Gould, 2015).

134 For the screening experiments, the day prior to transfection 9,000 HeLa cells expressing HA-

135 GLUT4-GFP were plated onto each well of a glass bottomed 96-well plate. Cells were

136 transfected with $3 \mu \mathrm{M}$ siRNA using the transfection reagent Lipofectamine 2000 and Opti-MEM

137 reduced serum medium according to the manufacturer's instructions. After $4 \mathrm{~h}$ exposure to

138 siRNA, growth media was added to each well and cells were incubated for $48 \mathrm{~h}$ prior to fixation;

139 cells were washed in PBS containing DAPI to visualise nuclei and representative fields of cells

140 (5 from each well, performed from 4 experimental replicates). Images were collected using a

$14163 \mathrm{x} / 1.4 \mathrm{NA}$ objective and analysed using ImageJ/FUJI software. Details of the siRNA sequences

142 employed are provided in Supplemental Table 1.

143 HA-GLUT4-GFP translocation: immunofluorescence - HeLa cells were washed three times in

144 ice-cold PBS then fixed in 3\% para-formaldehyde (PFA) for twenty min. After quenching and

145 washing in PBS containing 1\% BSA and 5\% goat serum, cells were incubated with anti-HA

146 monoclonal antibody (Covance Research Products t\# MMS 101P) at 1:500 for $45 \mathrm{~min}$. at room

147 temperature, then washed three times and surface bound monoclonal antibody detected using

148 Alex-Fluor labelled secondary antibodies (1:200). Cells were washed and mounted using

149 Immuno-mount and imaged using a Zeiss Pascal unit. Images were collected using a 63x/1.4NA

150 objective and analysed using ImageJ/FUJI software. Typically, between four and six random

151 fields of view were captured from each coverslip, and each experiment replicated four times on

152 independent passages of cells. Note that within a particular experiment, the intensity and pinhole

153 settings on the confocal were kept constant to allow direct comparison between experimental

154 conditions. Fluorescence intensity values from ImageJ were expressed as an HA/GFP ratio and

155 the value in unstimulated cells set $=1.0$ to allow comparison between independent experiments.

156 HA-GLUT4-GFP translocation: FACS - HA-GLUT4-GFP HeLa cells were seeded onto 6 well

157 plates $(\sim 300,000$ cells/well $) 24$ hours pre-analysis. On the experiment day cells were serum-

158 starved for two hours and half of the HA-GLUT4-GFP HeLa cell samples were stimulated with 1

$159 \mu \mathrm{M}$ insulin for $40 \mathrm{~min}$. at $37^{\circ} \mathrm{C}$. The plates were then placed on ice where all subsequent steps

160 were performed with use of ice-cold solutions. Surface GLUT4 was detected by immunostaining

161 with anti-HA antibodies in intact cells. Cells were incubated with labelling medium containing

162 primary anti-HA antibody at a concentration of 1:200 in DMEM with 10\% (v/v) FCS for one

163 hour. Cells were washed 3 times with PBS and incubated in labelling medium containing 1:300

164 secondary antibody conjugated with AlexaFluor ${ }^{\circledR} 647$ for one hour. Cells were washed with

165 PBS and gently dissociated with collagenase type I $(2 \mathrm{mg} / \mathrm{ml}(\mathrm{w} / \mathrm{v}))$ in PBS supplemented with

$1660.5 \mathrm{mM}$ EDTA and $10 \%(\mathrm{v} / \mathrm{v}) \mathrm{FCS}$ at $37^{\circ} \mathrm{C}$ for $10 \mathrm{~min}$. Samples were diluted in PBS and gently

167 filtered through a $100 \mu \mathrm{m}$ cell strainer to remove clumps of cells and analysed on a BD ${ }^{\mathrm{TM}}$ LSR II

168 flow cytometer. Events of 50,000 cells were collected for each experimental condition.

169 Identical methodology was employed when assaying translocation in 3T3-L1 adipocytes

170 expressing HA-GLUT4-GFP by FACS. In both cases, cells not expressing HA-GLUT4-GFP or

171 stained with HA-antibodies/secondary antibodies were used to determine background values.

172 Fluorescence intensities were calculated form geometric means.

173 Co-localisation assays - HA-GLUT4-GFP HeLa cells were cultured on 96 well plates with a

174 glass bottom. Prior to staining, they were washed 3x with PBS and fixed with PFA for $20 \mathrm{~min}$.

175 at room temperature. Cells were washed $3 \mathrm{x}$ with PBS and incubated in permeabilisation buffer 
176 (PBS containing $0.1 \% \mathrm{w} / \mathrm{v}$ Triton X100) for 4 min., then washed $3 \mathrm{x}$ with PBS and blocked with 177 PBS containing $0.2 \%(\mathrm{w} / \mathrm{v})$ fish skin gelatine and $0.1 \%(\mathrm{v} / \mathrm{v})$ goat serum for $30 \mathrm{~min}$. Cells were 178 stained by incubating with the primary antibody recognising a marker for the ER, the Golgi, or 179 the ERGIC for $60 \mathrm{~min}$. and with a secondary Alexa Fluor 568 tagged antibody for $45 \mathrm{~min}$.

180 Nucleus staining was carried out by incubation with $1 \mu \mathrm{g} / \mathrm{ml}$ DAPI for $5 \mathrm{~min}$. Representative 181 fields of cells (4 from duplicate coverslips for each condition, repeated four times) were imaged 182 using a Zeiss Pascal unit. Images were collected using a 63x/1.4NA objective and analysed 183 using ImageJ/Fiji software and the JaCoP plugin (Bolte and Cordelières, 2006).

184 Statistical analysis - Statistical analysis was conducted using Prism software; tests are described 185 in the figure legends or text.

186

\section{Results.}

188 GLUT4 in HeLa cells exhibits insulin-dependent translocation to the cell surface.

189 The majority of current investigations of GLUT4 recruitment to the plasma membrane suffer

190 from a low throughput due to the lengthy isolation and culturing of adipocytes, which are also

191 difficult to experimentally manipulate. As a result, there is a clear need for a robust insulin-

192

193

194 sensitive experimental cell model expressing GLUT4 as a useful 'test-tube' for initial experiments. Such a system would provide a platform for high throughput investigations. The HeLa cell line is an immortal cervical cancer cell line, originally isolated in 1951, and is the most widely investigated cell model (Macville et al., 1999). While HeLa cells do not contain any endogenous GLUT4, they do possess insulin sensitivity, and exhibit insulin-stimulated phosphorylation of Akt and AS160, two key signalling intermediates in insulin-stimulated GLUT4 translocation (Camus et al., 2020). We therefore generated stable clones of HeLa cells expressing HA-GLUT4-GFP. In the absence of insulin this construct was intracellularly sequestered and was present in intracellular peripheral vesicles and within a large perinuclear depot a distribution highly similar to that observed in 3T3-L1 adipocytes (Fig. 1). This well-characterised construct allows for detection of total GLUT4 levels (using the GFP signal) and the quantification of cell surface exposed molecules via the HA epitope inserted into the large exofacial domain between transmembrane helices I and II. This construct has been extensively validated in numerous laboratories (Lampson et al., 2000; Eyster, Duggins \& Olson, 2005; Eyster et al., 2006; Muretta, Romenskaia \& Mastick, 2008; Zhao et al., 2009; Lizunov et al., 2012). Figure 1 show the effect of insulin on HA-GLUT4-GFP translocation in HeLa cells (panel A) compared to 3T3-L1 adipocytes (panel B). In both cell types, a robust insulin-stimulated delivery of GLUT4 to the cell surface is observed. Figure 1A, B shows a typical set of confocal images in which the extent of translocation is revealed by the increase in HA staining upon insulin stimulation, and Fig. 1C shows the result of quantification of insulin-stimulated HA-GLUT4-GFP translocation using FACS. The latter allows quantification of many thousands of cells per condition and is the most reliable method for quantifying translocation in either cell type as a result. However, image analysis of fluorescence intensity of confocal images can also be readily used to quantify translocation - see further examples below. Insulin-stimulated translocation of HA-GLUT4GFP to the cell surface was inhibited robustly by $50 \mathrm{nM}$ wortmannin in both cell types (data not shown) (Clarke et al., 1994; Wang et al., 2019), suggesting similar insulin-dependent signalling processes underly these responses. Others have reported similar magnitudes of translocation using HeLa cells expressing HA-GLUT4-GFP across several studies of this type, insulin elicited 
221 a 2.5- to 3-fold increase in cell surface GLUT4 staining (Haga, Ishii \& Suzuki, 2011;

222 Kioumourtzoglou et al., 2015; Gulbranson et al., 2017; Wang et al., 2019). The roughly 3-fold

223 increase in GLUT4 translocation in HeLa cells is broadly comparable with other human tissues -

224 for a recent review of this issue see (Bryant \& Gould, 2020).

225

\section{Insulin-stimulated delivery of GLUT4 into the TIRF zone.}

227 Time-lapse live cell TIRFM was employed to quantify mobile and stationary vesicles located

228 adjacent to the plasma membrane following insulin stimulation in both cell types.

We first quantified the extent of translocation by measuring the time-dependent increase in GFP

230

231

232

233

234

235

236

237

238

239

240

241

242

243

244

245

246

247 signal in the TIRF zone (a typical data set for 3T3-L1 adipocytes is shown in Fig. 2A). Both analyses reveal that insulin stimulates translocation of HA-GLUT4-GFP to the surface, but that HeLa cells exhibit a smaller response than 3 T3-L1 adipocytes, $1.89 \pm 0.4$-fold versus $3.3 \pm 0.85$ fold. Note that the magnitude of the insulin response in these experiments is likely underestimated; quantification of the GFP signal does not represent only GLUT4 in the plasma membrane but will also report GLUT4 vesicles in the TIRF zone that are not fused with the plasma membrane. Figure 2B shows that the rate of translocation of GLUT4 in these cells exhibited half-times of $12.3 \pm 2.2 \mathrm{~min}$. in adipocytes ( $\mathrm{n}=15$ cells) and $17.1 \pm 6.3 \mathrm{~min}$. in HeLa cells $(\mathrm{n}=12)$. The value measured in 3T3-L1 adipocytes is somewhat slower than has been reported by others (5-10 min., see (Bogan, McKee \& Lodish, 2001; Govers, Coster \& James, 2004)). The slower rate of translocation in observed in our studies in 3T3-L1 adipocytes and HeLa cells may reflect a slower accumulation of total vesicles into the TIRF zone compared to levels of GLUT4 in the plasma membrane (Gibbs, Lienhard \& Gould, 1988; Subtil et al., 2000; Coster, Govers \& James, 2004; Martin, Lee \& McGraw, 2006; Gonzalez \& McGraw, 2006; Muretta, Romenskaia \& Mastick, 2008; Muretta \& Mastick, 2009; Xiong et al., 2010). This may also in part be a reflection of the temperature homeostasis on the stage being less than ideal due to the home-built nature of the incubation system; nevertheless, these data indicate that insulinstimulated translocation of GLUT4 in these cell types are broadly comparable.

\section{Comparison of GLUT4 vesicle movement near the plasma membrane.}

250

Stacks of time-lapse images were separated into 1-minute segments to determine the time-

251

252

253

254

255

256

257

258

259

260

261 dependent nature of vesicle dynamics as described in Materials and Methods. GLUT4containing vesicles were identified and the time dependent vesicle dynamics were compared for both cell lines. These values were measured for three individual cells within ten different 100 $\mu \mathrm{m}^{2}$ regions of interest across three separate platings of cells (Fig. 3A). In adipocytes, at the point of insulin stimulation, $t=0$, a notable increase in mobile GLUT4-containing vesicles was observed [note $\mathrm{t}=0$ constitutes the first $\sim 60$ seconds after insulin addition]. This period of increased mobility lasted $\sim 5 \mathrm{~min}$. before returning to a rate similar to that prior to insulin stimulation. This is in contrast to the behaviour of static GLUT4 vesicles. This is consistent with Fujita et al who observed an insulin-dependent increase in the number of rapidly moving GLUT4-containing vesicles approaching the plasma membrane in 3T3-L1 adipocytes, and an insulin-dependent increase in their tethering (Fujita et al., 2010).

262

263

264 Stenkula et al. report a dramatic increase in the rate of vesicle fusion with the plasma membrane between 1-5 min. post insulin (Stenkula et al., 2010; Lizunov et al., 2013a). The observed increase in dynamic GLUT4-containing vesicles over a similar time frame in this study would 
265 suggest vesicle activity in line with the previously presented kinetic model (Stenkula et al., 2010;

266 Lizunov et al., 2013a). The gradual increase in stationary GLUT4-containing vesicles was

267 predicted as vesicles continue to tether and fuse to the membrane upon stimulation (Fujita et al.,

268 2010), and several studies have reported an insulin-stimulated increase in vesicle tethering

269 (Lizunov et al., 2005, 2009, 2013b; Bai et al., 2007; Stenkula et al., 2010).

270 In HeLa cells, the quantity of mobile GLUT4-containing vesicles underwent a similar insulin-

271 dependent increase (Fig. 3B). The duration of this increased activity was observed to last $\sim 8$

272 min. after insulin stimulation. After the increase in activity the number of mobile GLUT4-

273 containing vesicles returned to a density of roughly 2 per $100 \mu \mathrm{m}^{2}$. The slightly longer duration

274 and lower final density is consistent with the extended $\boldsymbol{\tau}_{\mathbf{1} / \mathbf{2}}$ noted above for insulin-stimulated

275

276

277

278

279

280

281

282

283

284

285

286

287

288

289

290

291

292

293

294

295

296

297

298

299

300

301

302

303

304

305

306

307

308

309

GLUT4 translocation. One potentially interesting point of divergence between the two cell types is that the density of static GLUT4-containing vesicles decreased in response to insulin in HeLa cells (Figure 3B) whereas this remained static or even increased in 3T3-L1 adipocytes. This may explain the lower fold increase in GLUT4 translocation in HeLa cells. The explanation for this is not clear, but it is worth noting that the larger more rounded phenotype of 3T3-L1 adipocytes mean that the area of the cytoplasm sampled by TIRF is likely a smaller fraction than that sampled in HeLa cells. These observations support the hypothesis that behaviours of GLUT4containing vesicles differ subtly between different cell types, and that this may explain variations in the magnitude of GLUT4 translocation. Nevertheless, these data demonstrate that insulindependent mobilisation of GLUT4 to the cell surface is an inherent, common, property of a diverse array of cell types.

\section{Common trafficking pathways for GLUT4 in HeLa and 3T3-L1 adipocytes.}

Previous studies have established important roles for VAMP2 and VAMP4 in GLUT4 trafficking in adipocytes (Williams \& Pessin, 2008; Zhao et al., 2009; Sadler, Bryant \& Gould, 2015). VAMP2 plays an important role in insulin-dependent GLUT4 translocation to the cell surface, and VAMP4 in the delivery of newly synthesised GLUT4 into the intracellular GLUT4-storage vesicle compartment (Williams \& Pessin, 2008). To further test the validity of HeLa cells expressing HA-GLUT4-GFP as a model, we recapitulated the above experiments in this system. For this analysis, we quantified effects by measuring the fluorescence intensity of confocal images (see Materials and Methods), as FACS analysis was not practical for reasons of scale/cost. Representative confocal images and quantitation of these experiments are shown in Fig. 4 (blots of lysates from knockdown cells are shown in Supplemental Fig. 1).

We quantified the ability of insulin to increase plasma membrane GLUT4 levels after siRNA treatment, Fig. 4B, in which the basal (unstimulated levels) of plasma membrane GLUT4 are set at 1 . In these experiments scrambled siRNA treated cells exhibited a robust insulin response ( $\sim$-fold increase). Figure 4B shows that knockdown of VAMP2 significantly impaired insulinstimulated GLUT4 translocation to the cell surface. Consistent with published results (Williams \& Pessin, 2008) we observed no impairment of HA-GLUT4-GFP translocation in VAMP8 (Fig. 4A, B) or VAMP3, VAMP5 or VAMP7-depleted cells (Supplemental Fig. 2). However, we observed insulin-independent increases in HA-GLUT4-GFP levels in the plasma membrane upon VAMP2 and VAMP4 knockdown; this was larger following VAMP2 depletion than VAMP4 (2.7-fold versus 1.8-fold; Fig. 4C). VAMP2 knock down completely abolished any increase in GLUT4 in the plasma membrane following insulin stimulation. Conversely, following VAMP4 depletion, insulin still appeared to increase GLUT4 in the plasma membrane, although the

Peer] reviewing PDF | (2019:12:43873:1:1:NEW 31 Jan 2020) 
310 increase did not reach statistical significance (Fig. 4C; 1-7-fold, compared to $\sim 3$-fold in control

311 cells, $\mathrm{p}=0.18$ ). Direct comparison of our data with that in 3T3-L1 adipocytes is difficult, as

312 different methodologies have highlighted areas of contention. Williams and Pessin observed

313 reduced levels of cell surface GLUT4 in VAMP2-depleted cells, with no increase in basal levels

314 (Williams \& Pessin, 2008). Zhao et al report no effect of VAMP2 knockout on GLUT4

315 translocation (Zhao et al., 2009), but the preponderance of evidence argues that VAMP2 is the

316 key vSNARE for GLUT4 translocation (Bogan, 2012; Sadler, Bryant \& Gould, 2015; Klip,

317 McGraw \& James, 2019). It is interesting though that in the muscle cell line L6, tetanus toxin-

318 induced VAMP2 cleavage resulted in enhanced basal GLUT4 levels (Randhawa et al., 2000), a

319 result strikingly similar to that reported here.

320 These data might be taken to imply that in human cells, GLUT4 passes through a VAMP2-

321 dependent trafficking step, whereas not all GLUT4 passes through a VAMP4-dependent

322 trafficking step. This explanation is in line with the data of Williams and Pessin, who found that

323 VAMP4 was required for the sorting of newly synthesised GLUT4 into the GLUT4-storage

324 compartment (Williams \& Pessin, 2008). As VAMP2 and VAMP4 act at different trafficking

325 steps, depletion of either protein affects GLUT4 localisation differently. Consistent with this,

326 VAMP2 has been proposed to regulate GLUT4-containing vesicle fusion with the plasma

327 membrane and VAMP4 to regulate sorting into the GLUT4 storage compartment (Randhawa et

328 al., 2000; Williams \& Pessin, 2008; Sadler, Bryant \& Gould, 2015). However, the potential that

329 (for example) VAMP2 may regulate GLUT4 trafficking in other endosomal compartments, as

330 has been suggested for example for early to recycling endosome cargo traffic (Aikawa et al.,

331 2006; Ma \& Burd, 2019), is not unreasonable. The relative balance of different endosomal

332 pathways may differ between these cell types and thus reveal different facets of GLUT4

333 trafficking. Consistent with this, studies have suggested that GLUT4 trafficking differs between

334 human and murine cells (Camus et al., 2020), and hence some distinctions between these two

335 cell systems are not entirely unsurprising.

336 These data suggest that HeLa cells can offer clear insight into GLUT4 trafficking pathways in a

337 robust fashion. Using this system, rapid screening of the VAMP isoforms would have quickly focussed upon VAMP2 and VAMP4, isoforms which are known to contribute to GLUT4 trafficking in 3T3-L1 adipocytes (Williams \& Pessin, 2008). Such studies argue that HeLa cells can offer a reliable and rapid screening system for analysis of GLUT4 trafficking which can be used to focus on key targets for further studies in less tractable cell systems. We therefore attempted to perform such a screen.

\section{Identification of new components of GLUT4 trafficking.}

345 The use of RNAi screens has opened up new avenues of investigation (Simpson et al., 2007;

346 Simpson, 2009). siRNA in adipocytes, although achievable, is difficult, often requiring multiple rounds of transfection and is thus not easily transferred to larger screening platforms. Hence, we have examined the use of HeLa cells as a tool for screening using transfection of a library of siRNAs targeting a sub-set of SNARE proteins.

Recent studies have suggested that movement of GLUT4 from the ERGIC towards the GLUT4storage compartment plays a key role in the trafficking of newly synthesised GLUT4 in human cells (Camus et al., 2020). The SNARE proteins BET1, BET1L, GOSR1, GOSR2, SEC22A, SEC22B, SEC22C, Stx5, and YKT6 are known to be involved in trafficking to or from the ERGIC (Zhang and Hong, 2001; Appenzeller-Herzog and Hauri, 2006; Inoue, Tani and Tagaya, 
355

356

357

358

359

360

361

362

363

364

365

366

367

368

369

370

371

372

373

374

375

376

377

378

379

380

381

382

383

384

385

386

387

388

389

390

391

392

393

394

395

396

397

398

399

2016; Adnan et al., 2019; Linders et al., 2019). We therefore examined the effect of knockdown of these SNAREs on the distribution of HA-GLUT4-GFP by examining overlap of GFP with the ERGIC marker ERGIC-53 or the Golgi marker GM130. In addition, the GFP signal was used as an indication of GLUT4 levels. Details of the SMARTpools are provided in Supplemental Table 1. The results are shown in Fig. 5A-D. The subtle changes observed in these experiments highlight a major advantage in using HeLa cells. The ability to screen the required large numbers of cells allowed statistical significance to be established even for small changes in signal overlap. The plastic nature of membrane protein trafficking pathways predicates that interference with one pathway may be compensated for by another, resulting in subtle changes in steady-state localisation.

Knockdown of GOSR1 (GS28) selectively reduced the overlap of GLUT4 with ERGIC53; none of the other SNAREs examined gave any reproducible changes in overlap (Fig. 5B). GOSR1 participates in ER to Golgi traffic (Xu et al., 2002), but has also been implicated in the transport from early/recycling endosomes to the trans Golgi network (TGN) (Xu et al., 2002; Tai et al., 2004), in a SNARE complex containing Sx 5 and YKT6 where it mediates a trafficking route which functions in parallel with Sx16/Sx6 to mediate early/recycling endosome-TGN traffic. Strikingly, depletion of YKT6 enhanced the overlap of GLUT4 with GM130 (Fig. 5C). An important role for these SNAREs is also suggested by increased GLUT4 levels upon GOSR1 knockdown (Fig. 5D); it is important to note therefore that the reduction in ERGIC53 overlap may be underestimated as this analysis did not take into account total GLUT4 levels. These data, although preliminary, are interesting as they reveal a potential role of YKT6 and GOSR1 in GLUT4 trafficking.

Previous work from our group has implicated a Sx16/Sx6 enriched subdomain of the TGN in GLUT4 delivery into the GLUT4-storage compartment (Shewan et al., 2003; Proctor et al., 2006). Fig. 5 reveals that this siRNA analysis did not identify statistically significant effects of depletion of Sx6 or Sx16 on either distribution of levels of GLUT4. While both the overlap with ERGIC53 and the GLUT4/DAPI ratio were decreased upon Sx6 knockdown, these did not reach significance ( $p \sim 0.06$ in both cases). This may reflect the relative capacity of the GOSR1/YKT6 pathway compared to the $\mathrm{Sx} 6 / \mathrm{Sx} 16$ pathway in different cell types (Camus et al., 2020). These caveats notwithstanding, our data point to a role for the SNAREs GOSR1 and YKT6 in GLUT4 trafficking in human cells. Further work will be required to elaborate the specificity of these effects (is it only GLUT4 traffic that is perturbed?), the role of these SNAREs in more 'physiological' cells or tissues such as 3T3-L1 adipocytes where larger magnitudes of insulinresponsive trafficking can be studied. Nevertheless, this data clearly exemplifies the utility of HeLa cells in the rapid identification of potential targets for further study.

\section{Discussion.}

Here we characterise HA-GLUT4-GFP expressed in HeLa cells and show that these cells exhibit insulin-stimulated GLUT4 translocation with characteristics similar to those observed in 3T3-L1 adipocytes. We conclude that despite some differences between the two cells systems, such as that observed in the patterns of mobile and static vesicles in the TIRF zone, HeLa cells are a useful model for preliminary or high-throughput studies of GLUT4 traffic. Using this system, we confirm and extend previous studies revealing important roles for VAMP2 and VAMP4 in endosomal GLUT4 trafficking and identify GOSR1 and YKT6 as molecules that may control GLUT4 trafficking. 
400 Examination of GLUT4 vesicle dynamics in 3T3-L1 adipocytes have suggested the presence of 401 multiple different GLUT4-containing vesicles that are mobilised to the cell surface. Studies 402 suggest that GLUT4 traffic to the plasma membrane involves small, $60 \mathrm{~nm}$ diameter, GLUT4403 containing vesicles, and also at later times after insulin challenge, larger structures, thought to be 404 endosomal in origin ( 150nm) (Lizunov et al., 2005, 2013a; Xu et al., 2011; Chen et al., 2012). 405 Whether both structures exist in HeLa cells, and which contribute to (for example) the mobile 406 and static vesicles examined here remains to be ascertained. A distinction in the relative balance between these compartments in 3T3-L1 adipocytes and HeLa cells may account for the subtle distinctions observed in (for example) the behaviour of static vesicles and the effects of VAMP2 knockdown. Such distinctions though, should not detract from the utility of HeLa cells as a valid model of GLUT4 trafficking in human cells.

\section{Figure Legends.}

413 Figure 1. HA-GLUT4-GFP translocation in HeLa cells and 3T3-L1 adipocytes.

414 Panel A HeLa cells stably expressing HA-GLUT4-GFP were incubated without (Basal) or with $415100 \mathrm{nM}$ insulin for $1 \mathrm{~h}$ in serum-free media, washed fixed and stained for cell surface GLUT4 416 using the exofacial HA-epitope as described in Materials and Methods. Shown are 417 representative fields of cells in which the GFP moiety is pseudo-coloured green and the HA 418 staining is pseudo-coloured blue. A merged image is presented as shown. The data shown is 419 typical of more than 10 experiments of this type on different batches of stable HeLa cell clones 420 expressing HA-GLUT4-GFP.

421 Panel B 3T3-L1 cells stably expressing HA-GLUT4-GFP were incubated with or without 422 (Basal) $100 \mathrm{nM}$ insulin for $20 \mathrm{~min}$. in serum-free media, washed fixed and stained for cell surface GLUT4 using the exofacial HA-epitope as described in Materials and Methods. Shown are representative fields of cells exactly as outlined in Panel A. Data from a representative experiment is shown, replicated on four different batches of stable cell clones.

Panel C Translocation of HA-GLUT4-GFP to the cell surface in HeLa cells and 3T3-L1 adipocytes was assayed using FACS. Shown is the change in HA/GFP signal in response to insulin, expressed relative to the basal, in $n=3$ experiments for each of the cell types shown with 50,000 cells per condition. Statistical significance analysed by 2 -way ANOVA revealed a significant increase in cell surface GLUT4 levels in both cell types, ${ }^{*} \mathrm{p}<0.05$ and ${ }^{* *} \mathrm{p} \sim 0.01$.

\section{Figure 2. Translocation of HA-GLUT4-GFP assayed by TIRFM.}

433 HA-GLUT4-GFP expressing 3T3-L1 adipocytes were serum-starved for $2 \mathrm{~h}$ and mounted on a heated stage in a home-built TIRF system. Images corresponding to GFP fluorescence were collected prior to insulin addition $(0 \mathrm{~min}$.) then at the indicated times after addition of $100 \mathrm{nM}$ insulin. Scale bar $-20 \mu \mathrm{m}$. Data from a representative experiment is shown in Panel A. Panel B shows quantification of the time course of insulin-stimulated increase in GFP fluorescence in the TIRF zone in either HeLa or 3T3-L1 adipocytes. Panel $\mathbf{C}$ shows the magnitude of the increase in GFP signal in the TIRF zone upon exposure of the cells to 100nM insulin. For both Panels $\mathbf{B}$ and $\mathbf{C}$, the data is the mean \pm SEM of 12 HeLa cells and 15 3T3-L1 cells imaged at each time point from at least three biological replicates. * Statistically significant compared to 
444 Figure 3. Quantification of vesicle dynamics in the TIRF zone.

445 Counts of mobile and stationary vesicles for 3T3-L1 adipocytes and HeLa cells stimulated by $446100 \mathrm{nM}$ insulin were determined as outlined in Materials and Methods. 10 individual $100 \mu \mathrm{m}^{2}$ 447 regions of interest were analysed from 3 cells from three separate platings of cells. Error bars 448 correspond to standard deviation for 30 measured ROIs. Images were recorded at a frame rate of 449 $2 \mathrm{~Hz}$ for $15 \mathrm{~min}$. where time point 0 corresponds to point of insulin addition.

450

Figure 4. The effect of knockdown of VAMP isoforms on HA-GLUT4-GFP translocation.

452

453

454

455

456

457

458

459

460

461

462

463

464

465

466

467

468

469

470

471

472

473

474

475

476

477

478

479

480

481

482

483

484

485

486

HeLa cells expressing HA-GLUT4-GFP were grown and transfected with 200nM scrambled control sequence (SCR), VAMP2, 4 or 8 SMARTpool siRNA as described. Cells were serumstarved (basal) before being treated with or without $1 \mu \mathrm{M}$ insulin (insulin) for $20 \mathrm{~min}$.. Cells were fixed and surface HA was stained as described. Panel A shows immunofluorescence images of a typical field for each condition are shown. Panel B shows the fold-change in the HA/GFP ratio with insulin-stimulation. Values were compared using Student's t-test $\left({ }^{*} p<0.05\right.$, $* * p>0.05)$ and are means \pm SD of 16 random fields of view, taken from 4 independent experiments. In this analysis, the HA/GFP ratio in the absence of insulin is set equal to 1 for each siRNA. VAMP2 knockdown significantly inhibited insulin-simulated HA-GLUT4-GFP translocation, $* p=0.01$. The apparent reduction in translocation upon VAMP4 knockdown did not reach statistical significance. Panel $\mathbf{C}$ quantifies the basal HA/GFP ratio for each VAMP knockdown compared to that observed in SCR siRNA treated cells; values represent the means \pm SD of 16 fields of view, taken from 4 independent experiments. Statistical significance analysed by 2-way ANOVA, ** $<<0.05$.

\section{Figure 5. GLUT4 trafficking involves GOSR1 and Ykt6.}

HeLa cells expressing HA-GLUT4-GFP were grown and transfected with 200nM scrambled control sequence (-VE CON) or SMARTpool siRNA targeting the indicated SNARE proteins. $48 \mathrm{~h}$ later, cells were fixed and stained with organelle-specific antibodies (shown in Panel A is a stain for the Golgi resident GM130 as a representative example). The extent of overlap between the GFP (GLUT4) signal and ERGIC53 or GM130 was quantified from 5 random fields each containing 8-12 cells from 4 independent experiments as described in Materials and Methods. Shown in Panel B is quantification of the ERGIC53/GLUT4 overlap and in Panel C the GM130/GLUT4 overlap. In Panel D, an estimate of total GLUT4 levels was determined by quantification of the GFP/DAPI ratio from the same experimental datasets. In each of Panels B, $\mathbf{C}$ and $\mathbf{D}$, data of all four experiments was normalised to the negative control experiment and 2way ANOVA with $95 \%$ confidence intervals was used for statistical analysis. GOSR1: $\mathrm{p}=0.0115$ (panel B). Ykt6: $\mathrm{p}=0.0036$ (panel C). GOSR1: $\mathrm{p}<0.0001$ (panel D).

\section{Supplemental Figure 1}

HA-GLUT4-GFP expressing HeLa cells were transfected with the indicated siRNA SmartPool $(\mathrm{SCR}=$ scrambled control) and lysates prepared as described in Materials and Methods. Equal amounts of lysate were separate on SDS-PAGE gels and immunoblotted for levels of the proteins indicated on the left of the figure. VAMP2 levels were decreased by $55+18 \%$ and VAMP4 levels decreased $82+9 \%$ in $n=4$ experiments of this type. 
488 Supplemental Figure 2.

489 HeLa cells expressing HA-GLUT4-GFP were grown and transfected with 200nM scrambled 490 control sequence (Scr), VAMP3, 5 or 7 SMARTpool siRNA as described. Cells were serum491 starved (basal) before being treated with or without $1 \mu \mathrm{M}$ insulin (insulin) for $20 \mathrm{~min}$. and cell 492 surface HA staining quantified as outlined in Materials and Methods. Values shown are means \pm 493 SD of 16 random fields of view, taken from 4 independent experiments. In each case, insulin 494 induced a statistically significant increase in cell surface GLUT4 staining, but Basal

495 (unstimulated) or insulin-stimulated values did not differ significantly from Scr-treated cells for 496 any of the VAMP knockdowns shown.

497

498 
499

500

501

502

503

504

505

506

507

508

509

510

511

512

513

514

515

516

517

518

519

520

521

522

523

524

525

526

527

528

529

530

531

532

533

534

535

536

537

538

539

540

541

542

543

544

\section{Bibliography}

Aikawa Y., Lynch KL., Boswell KL., Martin TFJ. 2006. A second SNARE role for exocytic SNAP25 in endosome fusion. Molecular Biology of the Cell 17:2113-2124.

Bai L., Wang Y., Fan J., Chen Y., Ji W., Qu A., Xu P., James DE., Xu T. 2007. Dissecting multiple steps of GLUT4 trafficking and identifying the sites of insulin action. Cell Metabolism 5:47-57.

Bogan JS. 2012. Regulation of glucose transporter translocation in health and diabetes. Annual Review of Biochemistry 81:507-532.

Bogan JS., McKee AE., Lodish HF. 2001. Insulin-responsive compartments containing GLUT4 in 3T3-L1 and CHO cells: regulation by amino acid concentrations. Molecular and Cellular Biology 21:4785-4806.

Bryant NJ., Gould GW. 2020. Insulin Stimulated GLUT4 translocation - size isn't everything! Current Opinion in Cell Biology submitted.

Bryant NJ., Govers R., James DE. 2002. Regulated trafficking of the glucose transporter, Glut4. Nat. Rev. Mol. Cell Biol. 3:267-277.

Camus,SM., Camus MD., Figueras-Novoa C., Boncompain G., Sadacca LA., Esk C., Bigot A., Gould GW., Kioumourtzoglou D., Perez F., Bryant NJ., Mukherjee S., Brodsky FM. CHC22 clathrin mediates traffic from early secretory compartments for human GLUT4 pathway biogenesis. The Journal of Cell Biology (2020) 219, pii: e201812135. doi: 10.1083/jcb.201812135.

Chen Y., Wang Y., Zhang J., Deng Y., Jiang L., Song E., Wu XS., Hammer JA., Xu T., Lippincott-Schwartz J. 2012. Rab10 and myosin-Va mediate insulin-stimulated GLUT4 storage vesicle translocation in adipocytes. The Journal of Cell Biology 198:545-560.

Clarke JF., Young PW., Yonezawa K., Kasuga M., Holman GD. 1994. Inhibition of the translocation of GLUT1 and GLUT4 in 3T3-L1 cells by the phosphatidylinositol 3-kinase inhibitor, wortmannin. The Biochemical Journal 300 ( Pt 3):631-635.

Coster ACF., Govers R., James DE. 2004. Insulin stimulates the entry of GLUT4 into the endosomal recycling pathway by a quantal mechanism. Traffic 5:763-771.

Eyster CA., Duggins QS., Gorbsky GJ., Olson AL. 2006. Microtubule network is required for insulin signaling through activation of Akt/protein kinase B: evidence that insulin stimulates vesicle docking/fusion but not intracellular mobility. The Journal of Biological Chemistry 281:39719-39727.

Eyster CA., Duggins QS., Olson AL. 2005. Expression of constitutively active Akt/protein kinase B signals GLUT4 translocation in the absence of an intact actin cytoskeleton. The Journal of Biological Chemistry 280:17978-17985.

Fujita H., Hatakeyama H., Watanabe TM., Sato M., Higuchi H., Kanzaki M. 2010. Identification of three distinct functional sites of insulin-mediated GLUT4 trafficking in adipocytes using quantitative single molecule imaging. Mol Biol Cell 21:2721-31.

Garvey WT., Maianu L., Zhu JH., Brechtel-Hook G., Wallace P., Baron AD. 1998. Evidence for defects in the trafficking and translocation of GLUT4 glucose transporters in skeletal muscle as a cause of human insulin resistance. The Journal of Clinical Investigation 101:2377-2386.

Garvey WT., Maianu L., Zhu JH., Hancock JA., Golichowski AM. 1993. Multiple defects in the adipocyte glucose transport system cause cellular insulin resistance in gestational diabetes. Diabetes 42:1773-1785.

Gibbs EM., Lienhard GE., Gould GW. 1988. Insulin-induced translocation of glucose

Peer) reviewing PDF | (2019:12:43873:1:1:NEW 31 Jan 2020) 
545

552

553

554

555

556

557

558

559

560

561

562

563

564

565

566

567

568

569

570

571

572

573

574

575

576

577

578

579

580

581

582

583

584

585

586

587

588

589

590

transporters to the plasma membrane precedes full stimulation of hexose transport. Biochemistry 27:6681-6685.

Gonzalez E., McGraw TE. 2006. Insulin signaling diverges into Akt-dependent and -independent signals to regulate the recruitment/docking and the fusion of GLUT4 vesicles to the plasma membrane. Molecular Biology of the Cell 17:4484-4493.

Govers R., Coster ACF., James DE. 2004. Insulin increases cell surface GLUT4 levels by dose dependently discharging GLUT4 into a cell surface recycling pathway. Molecular and Cellular Biology 24:6456-6466.

Gulbranson DR., Davis EM., Demmitt BA., Ouyang Y., Ye Y., Yu H., Shen J. 2017. RABIF/MSS4 is a Rab-stabilizing holdase chaperone required for GLUT4 exocytosis. Proceedings of the National Academy of Sciences of the United States of America 114:E8224-E8233.

Haga Y., Ishii K., Suzuki T. 2011. N-glycosylation is critical for the stability and intracellular trafficking of glucose transporter GLUT4. The Journal of Biological Chemistry 286:31320-31327.

Herbert AD., Carr AM., Hoffmann E. 2014. FindFoci: a focus detection algorithm with automated parameter training that closely matches human assignments, reduces human inconsistencies and increases speed of analysis. Plos One 9:e114749.

Jaldin-Fincati JR., Pavarotti M., Frendo-Cumbo S., Bilan PJ., Klip A. 2017. Update on GLUT4 vesicle traffic: A cornerstone of insulin action. Trends in Endocrinology and Metabolism 28:597-611.

Kahn BB. 1992. Alterations in glucose transporter expression and function in diabetes: mechanisms for insulin resistance. Journal of Cellular Biochemistry 48:122-128.

Kawase K., Nakamura T., Takaya A., Aoki K., Namikawa K., Kiyama H., Inagaki S., Takemoto H., Saltiel AR., Matsuda M. 2006. GTP hydrolysis by the Rho family GTPase TC10 promotes exocytic vesicle fusion. Developmental Cell 11:411-421.

Kioumourtzoglou D., Pryor PR., Gould GW., Bryant NJ. 2015. Alternative routes to the cell surface underpin insulin-regulated membrane trafficking of GLUT4. Journal of Cell Science 128:2423-2429.

Klip A., McGraw TE., James DE. 2019. Thirty sweet years of GLUT4. The Journal of Biological Chemistry 294:11369-11381.

Lampson MA., Racz A., Cushman SW., McGraw TE. 2000. Demonstration of insulin-responsive trafficking of GLUT4 and vpTR in fibroblasts. Journal of Cell Science 113 ( Pt 22):4065-4076.

Lizunov VA., Lee J-P., Skarulis MC., Zimmerberg J., Cushman SW., Stenkula KG. 2013 a. Impaired tethering and fusion of GLUT4 vesicles in insulin-resistant human adipose cells. Diabetes 62:3114-3119.

Lizunov VA., Lisinski I., Stenkula K., Zimmerberg J., Cushman SW. 2009. Insulin regulates fusion of GLUT4 vesicles independent of Exo70-mediated tethering. The Journal of Biological Chemistry 284:7914-7919.

Lizunov VA., Matsumoto H., Zimmerberg J., Cushman SW., Frolov VA. 2005. Insulin stimulates the halting, tethering, and fusion of mobile GLUT4 vesicles in rat adipose cells. The Journal of Cell Biology 169:481-489.

Lizunov VA., Stenkula KG., Lisinski I., Gavrilova O., Yver DR., Chadt A., Al-Hasani H., Zimmerberg J., Cushman SW. 2012. Insulin stimulates fusion, but not tethering, of GLUT4 vesicles in skeletal muscle of HA-GLUT4-GFP transgenic mice. American 
591

592

593

594

595

596

597

598

599

600

601

602

603

604

605

606

607

608

609

610

611

612

613

614

615

616

617

618

619

620

621

622

623

624

625

626

627

628

629

630

631

632

633

634

635

636

Journal of Physiology. Endocrinology and Metabolism 302:E950-60.

Lizunov VA., Stenkula K., Troy A., Cushman SW., Zimmerberg J. 2013b. Insulin regulates Glut4 confinement in plasma membrane clusters in adipose cells. Plos One 8:e57559.

Ma M., Burd CG. 2019. Retrograde trafficking and quality control of yeast synaptobrevin, Snc1, are conferred by its transmembrane domain. Molecular Biology of the Cell 30:17291742.

Macville M., Schröck E., Padilla-Nash H., Keck C., Ghadimi BM., Zimonjic D., Popescu N., Ried T. 1999. Comprehensive and definitive molecular cytogenetic characterization of HeLa cells by spectral karyotyping. Cancer Research 59:141-150.

Martin OJ., Lee A., McGraw TE. 2006. GLUT4 distribution between the plasma membrane and the intracellular compartments is maintained by an insulin-modulated bipartite dynamic mechanism. The Journal of Biological Chemistry 281:484-490.

Muretta JM., Mastick CC. 2009. How insulin regulates glucose transport in adipocytes. Vitam Horm 80:245-86.

Muretta JM., Romenskaia I., Mastick CC. 2008. Insulin releases Glut4 from static storage compartments into cycling endosomes and increases the rate constant for Glut4 exocytosis. The Journal of Biological Chemistry 283:311-323.

Orlicky DJ., Schaack J. 2001. Adenovirus transduction of 3T3-L1 cells. Journal of Lipid Research 42:460-466.

Proctor KM., Miller SCM., Bryant NJ., Gould GW. 2006. Syntaxin 16 controls the intracellular sequestration of GLUT4 in 3T3-L1 adipocytes. Biochemical and Biophysical Research Communications 347:433-438.

Puri V., Chakladar A., Virbasius JV., Konda S., Powelka AM., Chouinard M., Hagan GN., Perugini R., Czech MP. 2007. RNAi-based gene silencing in primary mouse and human adipose tissues. Journal of Lipid Research 48:465-471.

Randhawa VK., Bilan PJ., Khayat ZA., Daneman N., Liu Z., Ramlal T., Volchuk A., Peng XR., Coppola T., Regazzi R., Trimble WS., Klip, A. 2000. VAMP2, but not VAMP3/cellubrevin, mediates insulin-dependent incorporation of GLUT4 into the plasma membrane of L6 myoblasts. Molecular Biology of the Cell 11:2403-2417.

Sadler JBA., Bryant NJ., Gould GW. 2015. Characterization of VAMP isoforms in 3T3-L1 adipocytes: implications for GLUT4 trafficking. Molecular Biology of the Cell 26:530536.

Shewan AM., van Dam EM., Martin S., Luen TB., Hong W., Bryant NJ., James DE. 2003. GLUT4 recycles via a trans-Golgi network (TGN) subdomain enriched in Syntaxins 6 and 16 but not TGN38: involvement of an acidic targeting motif. Molecular Biology of the Cell 14:973-986.

Simpson JC. 2009. Screening the secretion machinery: High throughput imaging approaches to elucidate the secretory pathway. Seminars in Cell \& Developmental Biology 20:903-909.

Simpson JC., Cetin C., Erfle H., Joggerst B., Liebel U., Ellenberg J., Pepperkok R. 2007. An RNAi screening platform to identify secretion machinery in mammalian cells. Journal of Biotechnology 129:352-365.

Stenkula KG., Lizunov VA., Cushman SW., Zimmerberg J. 2010. Insulin controls the spatial distribution of GLUT4 on the cell surface through regulation of its postfusion dispersal. Cell Metabolism 12:250-259.

Sternberg. 1983. Biomedical Image Processing. Computer 16:22-34.

Subtil A., Lampson MA., Keller SR., McGraw TE. 2000. Characterization of the insulin-

PeerJ reviewing PDF | (2019:12:43873:1:1:NEW 31 Jan 2020) 
637

638

639

640

641

642

643

644

645

646

647

648

649

650

651

652

653

654

655

656

657

658

659

660 regulated endocytic recycling mechanism in 3T3-L1 adipocytes using a novel reporter molecule. The Journal of Biological Chemistry 275:4787-4795.

Tai G., Lu L., Wang TL., Tang BL., Goud B., Johannes L., Hong W. 2004. Participation of the syntaxin 5/Ykt6/GS28/GS15 SNARE complex in transport from the early/recycling endosome to the. Mol. Biol. Cell 15:4011-4022.

Wang S., Crisman L., Miller J., Datta I., Gulbranson DR., Tian Y., Yin Q., Yu H., Shen J. 2019. Inducible Exoc7/Exo70 knockout reveals a critical role of the exocyst in insulin-regulated GLUT4 exocytosis. The Journal of Biological Chemistry.

Williams D., Pessin JE. 2008. Mapping of R-SNARE function at distinct intracellular GLUT4 trafficking steps in adipocytes. The Journal of Cell Biology 180:375-387.

Xiong W., Jordens I., Gonzalez E., McGraw TE. 2010. GLUT4 is sorted to vesicles whose accumulation beneath and insertion into the plasma membrane are differentially regulated by insulin and selectively affected by insulin resistance. Molecular Biology of the Cell 21:1375-1386.

Xu Y., Martin S., James DE., Hong W. 2002. GS15 forms a SNARE complex with syntaxin 5, GS28, and Ykt6 and is implicated in traffic in the early cisternae of the Golgi apparatus. Molecular Biology of the Cell 13:3493-3507.

Xu Y., Rubin BR., Orme CM., Karpikov A., Yu C., Bogan JS., Toomre DK. 2011. Dual-mode of insulin action controls GLUT4 vesicle exocytosis. The Journal of Cell Biology 193:643653.

Zhao P., Yang L., Lopez JA., Fan J., Burchfield JG., Bai L., Hong W., Xu T., James DE. 2009. Variations in the requirement for v-SNAREs in GLUT4 trafficking in adipocytes. Journal of Cell Science 122:3472-3480. 


\section{Figure 1}

HA-GLUT4-GFP translocation in HeLa cells and 3T3-L1 adipocytes.

Panel A HeLa cells stably expressing HA-GLUT4-GFP were incubated without (Basal) or with $100 \mathrm{nM}$ insulin for $1 \mathrm{~h}$ in serum-free media, washed fixed and stained for cell surface GLUT4 using the exofacial HA-epitope as described in Materials and Methods. Shown are representative fields of cells in which the GFP moiety is pseudo-coloured green and the HA staining is pseudo-coloured blue. A merged image is presented as shown. The data shown is typical of more than 10 experiments of this type on different batches of stable HeLa cell clones expressing HA-GLUT4-GFP.Panel B 3T3-L1 cells stably expressing HA-GLUT4-GFP were incubated with or without (Basal) $100 \mathrm{nM}$ insulin for 20 minutes in serum-free media, washed fixed and stained for cell surface GLUT4 using the exofacial HA-epitope as described in Materials and Methods. Shown are representative fields of cells exactly as outlined in Panel A. Data from a representative experiment is shown, replicated on four different batches of stable cell clones.Panel C Translocation of HA-GLUT4-GFP to the cell surface in HeLa cells and 3T3-L1 adipocytes was assayed using FACS. Shown is the change in HA/GFP signal in response to insulin, expressed relative to the basal, in $n=3$ experiments for each of the cell types shown with 50,000 cells per condition. A significant increase in cell surface GLUT4 levels was detected in both cell types, $* p<0.05$ and ${ }^{* *} p \sim 0.01$. 

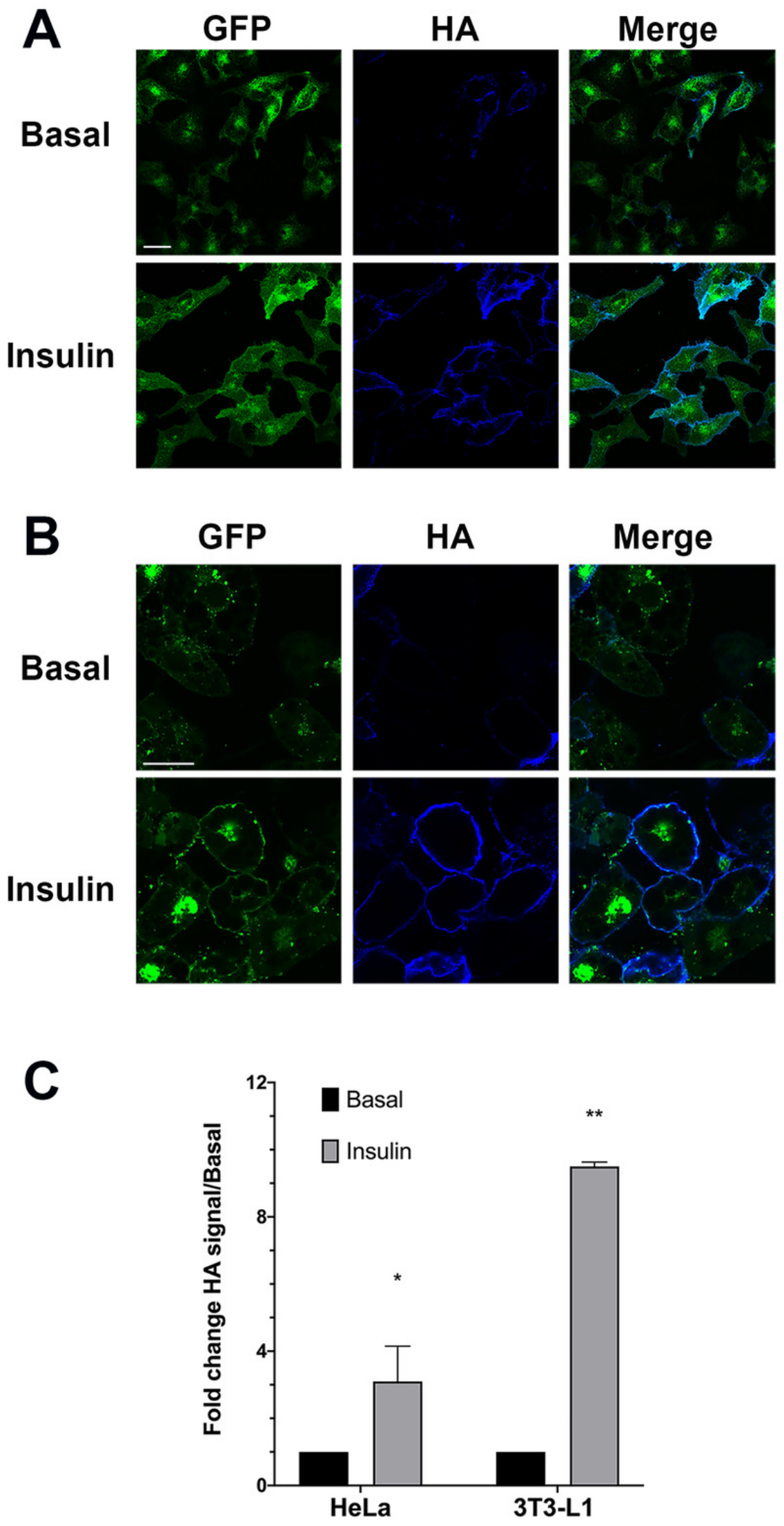


\section{Figure 2}

Translocation of HA-GLUT4-GFP assayed by TIRFM

HA-GLUT4-GFP expressing 3T3-L1 adipocytes were serum-starved for $2 \mathrm{~h}$ and mounted on a heated stage in a home-built TIRF system. Images corresponding to GFP fluorescence were collected prior to insulin addition ( 0 min.) then at the indicated times after addition of $100 \mathrm{nM}$ insulin. Scale bar - $20 \mu \mathrm{m}$. Data from a representative experiment is shown in Panel A. Panel B shows quantification of the time course of insulin-stimulated increase in GFP fluorescence in the TIRF zone in either HeLa or 3T3-L1 adipocytes. Panel C shows the magnitude of the increase in GFP signal in the TIRF zone upon exposure of the cells to 100nM insulin. For both Panels B and C, the data is the mean + SEM of 12 HeLa cells and 15 3T3-L1 cells imaged at each time point from at least three biological replicates. *Statistically significant compared to basal $p=0.05$; $* * p=0.01$ Statistical significance analysed by 2 -way ANOVA. 

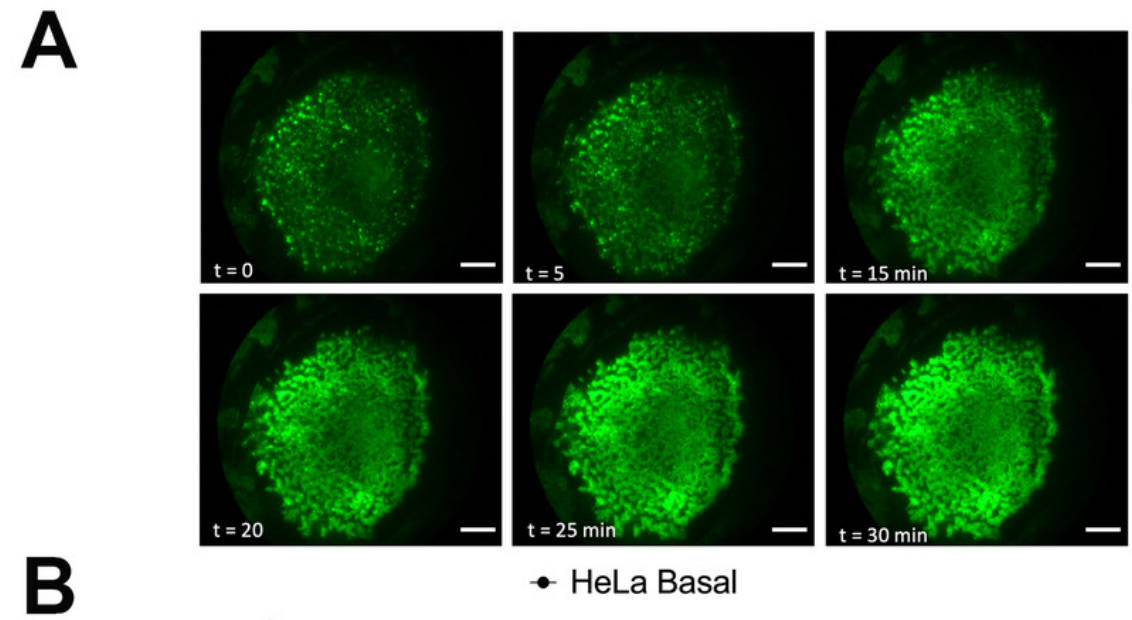

- HeLa Basal

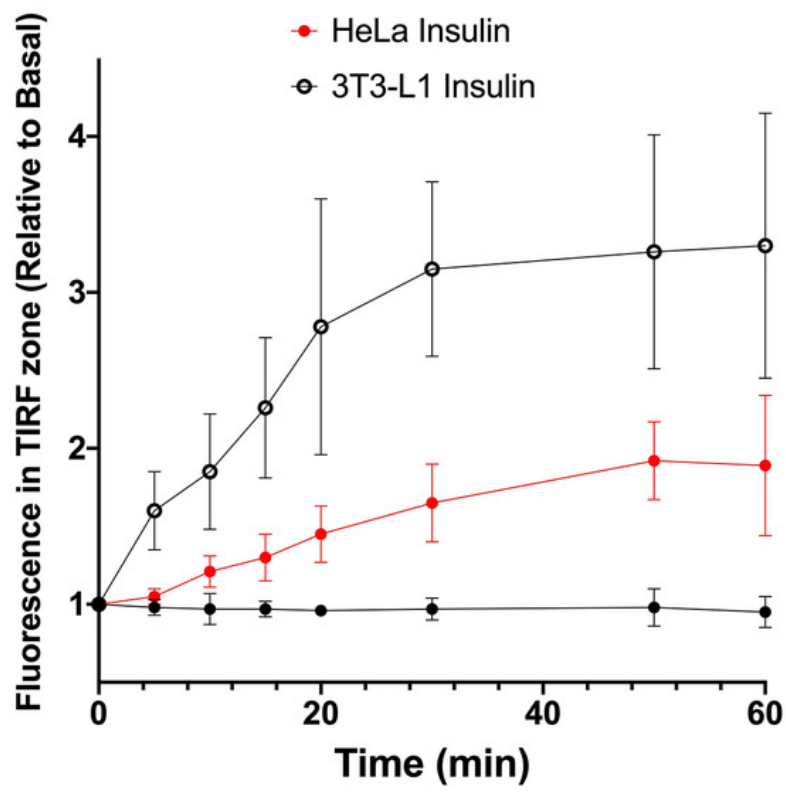

C

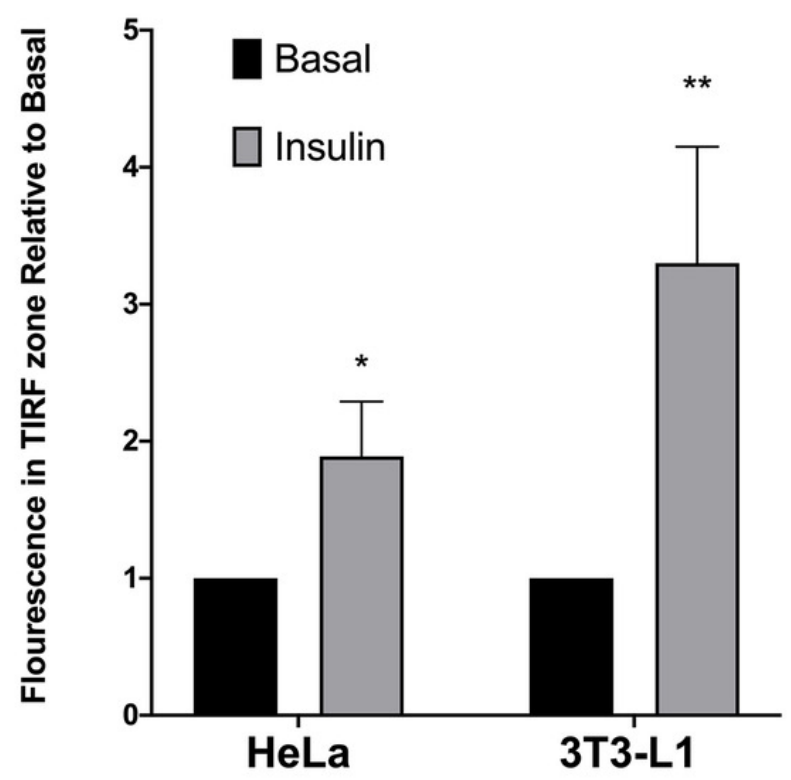




\section{Figure 3}

Quantification of vesicle dynamics in the TIRF zone.

Counts of mobile and stationary vesicles for 3T3-L1 adipocytes and HeLa cells stimulated by $100 \mathrm{nM}$ insulin were determined as outlined in Materials and Methods. 10 individual $100 \mu \mathrm{m}^{2}$ regions of interest were analysed from 3 cells from three separate platings of cells. Error bars correspond to standard deviation for 30 measured ROIs. Images were recorded at a frame rate of $2 \mathrm{~Hz}$ for 15 minutes where time point 0 corresponds to point of insulin addition. 
A

- Static GLUT4 vesicles

- Mobile GLUT4 vesicles
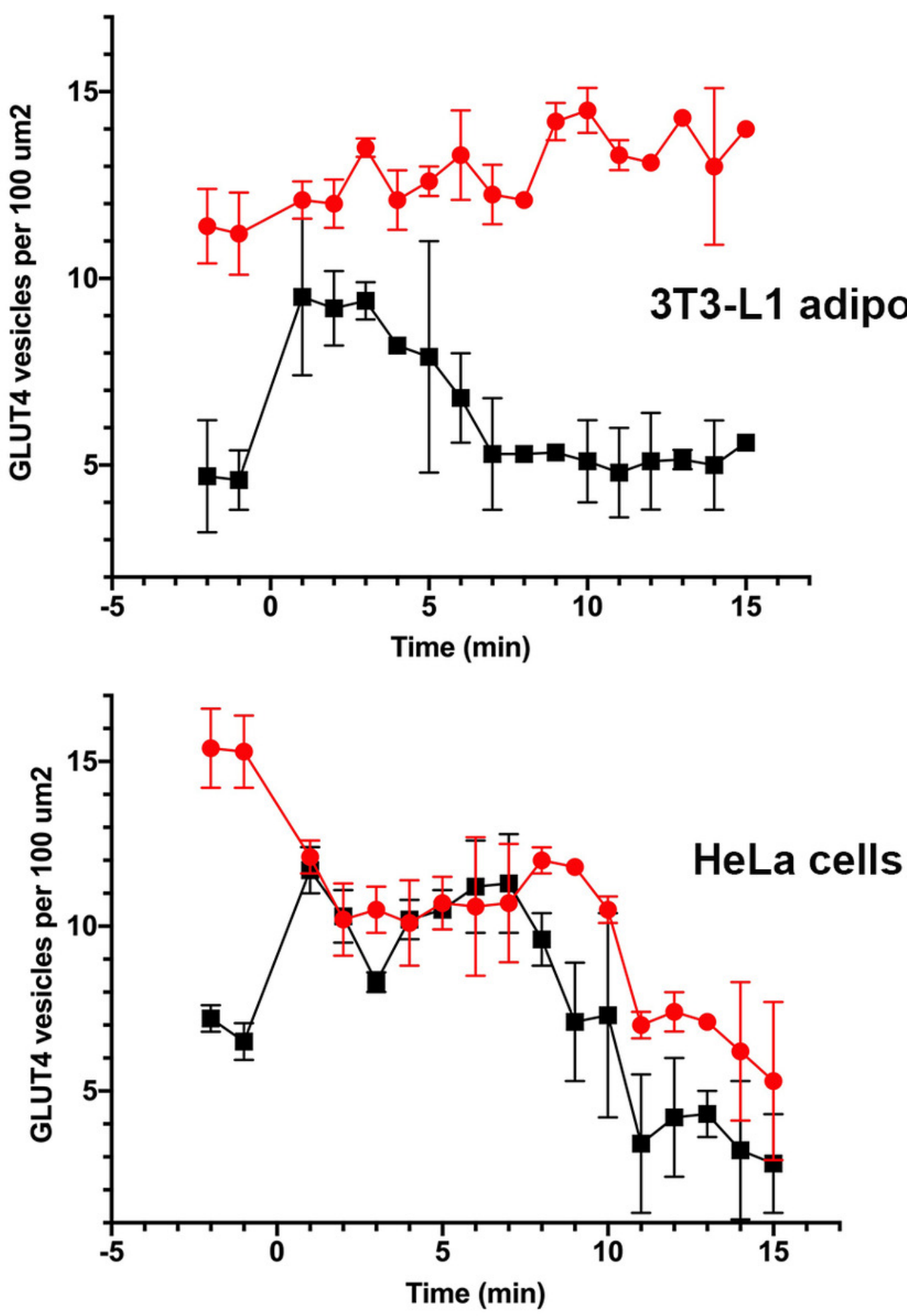


\section{Figure 4}

The effect of knockdown of VAMP isoforms on HA-GLUT4-GFP translocation.

HeLa cells expressing HA-GLUT4-GFP were grown and transfected with 200nM scrambled control sequence (SCR), VAMP2, 4 or 8 SMARTpool siRNA as described. Cells were serumstarved (basal) before being treated with or without $1 \mu \mathrm{M}$ insulin (insulin) for 20 minutes. Cells were fixed and surface HA was stained as described. Panel A shows immunofluorescence images of a typical field for each condition are shown. Panel B shows the fold-change in the HA/GFP ratio with insulin-stimulation. Values were compared using Student's t-test $\left({ }^{*} p<0.05\right.$, $\left.{ }^{* *} p>0.05\right)$ and are means \pm SD of 16 random fields of view, taken from 4 independent experiments. In this analysis, the HA/GFP ratio in the absence of insulin is set equal to 1 for each siRNA. VAMP2 knockdown significantly inhibited insulin-simulated HA-GLUT4-GFP translocation, $* p=0.01$. The apparent reduction in translocation upon VAMP4 knockdown did not reach statistical significance. Panel $\mathbf{C}$ quantifies the basal HA/GFP ratio for each VAMP knockdown compared to that observed in SCR siRNA treated cells; values represent the means \pm SD of 16 fields of view, taken from 4 independent experiments. $* * p<0.05$. 


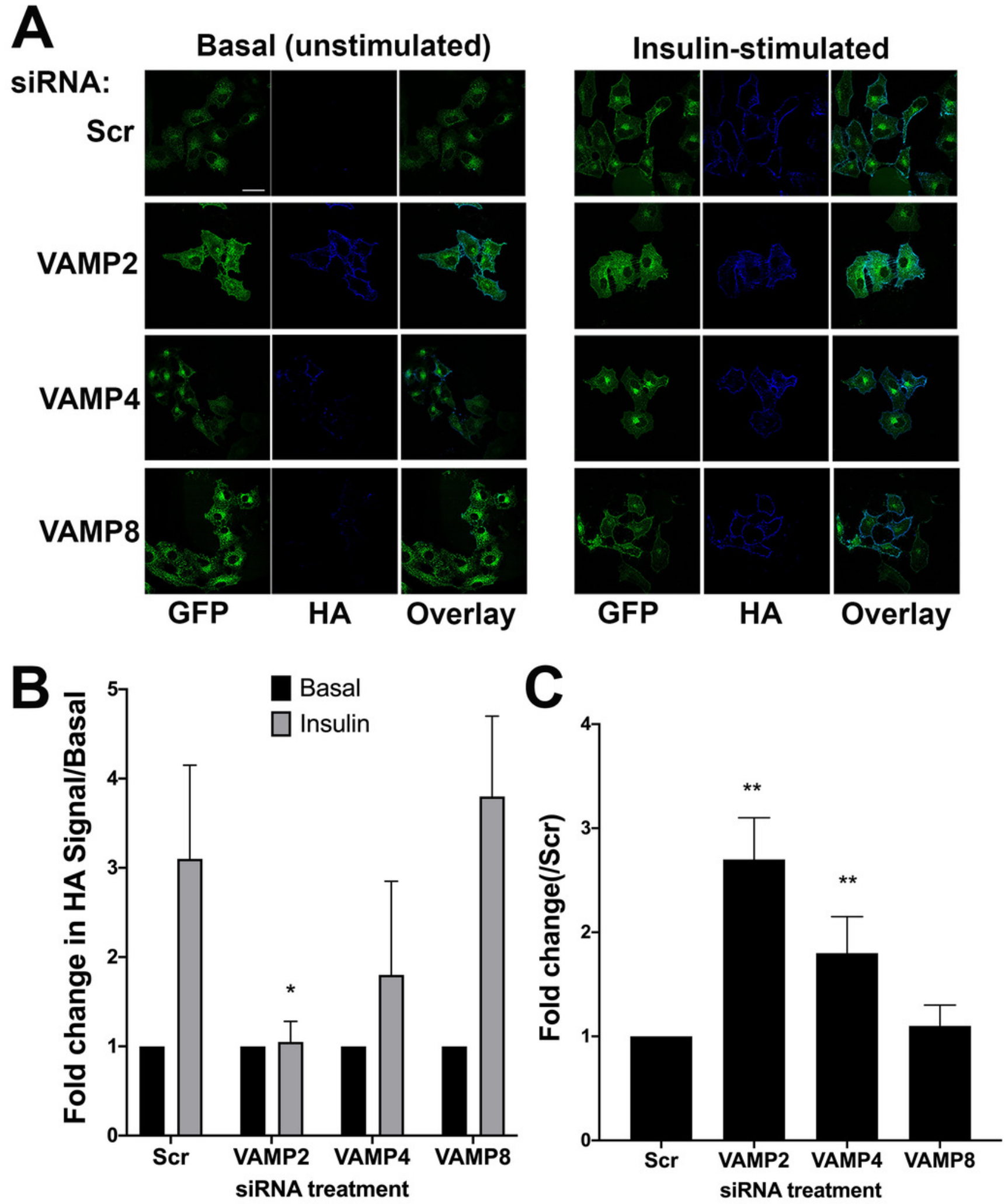




\section{Figure 5}

GLUT4 trafficking involves GOSR1 and Ykt6.

HeLa cells expressing HA-GLUT4-GFP were grown and transfected with 200nM scrambled control sequence (-VE CON) or SMARTpool siRNA targeting the indicated SNARE proteins. 48h later, cells were fixed and stained with organelle-specific antibodies (shown in Panel A is a stain for the Golgi resident GM130 as a representative example). The extent of overlap between the GFP (GLUT4) signal and ERGIC53 or GM130 was quantified from 5 random fields each containing 8-12 cells from 4 independent experiments as described in Materials and Methods. Shown in Panel B is quantification of the ERGIC53/GLUT4 overlap and in Panel C the GM130/GLUT4 overlap. In Panel D, an estimate of total GLUT4 levels was determined by quantification of the GFP/DAPI ratio from the same experimental datasets. In each of Panels B, C and D, data of all four experiments was normalised to the negative control experiment and 2-way ANOVA with $95 \%$ confidence intervals was used for statistical analysis. GOSR1: $p$ $=0.0115$ (panel B). Ykt6: $p=0.0036$ (panel C). GOSR1: $p<0.0001$ (panel D). 
A

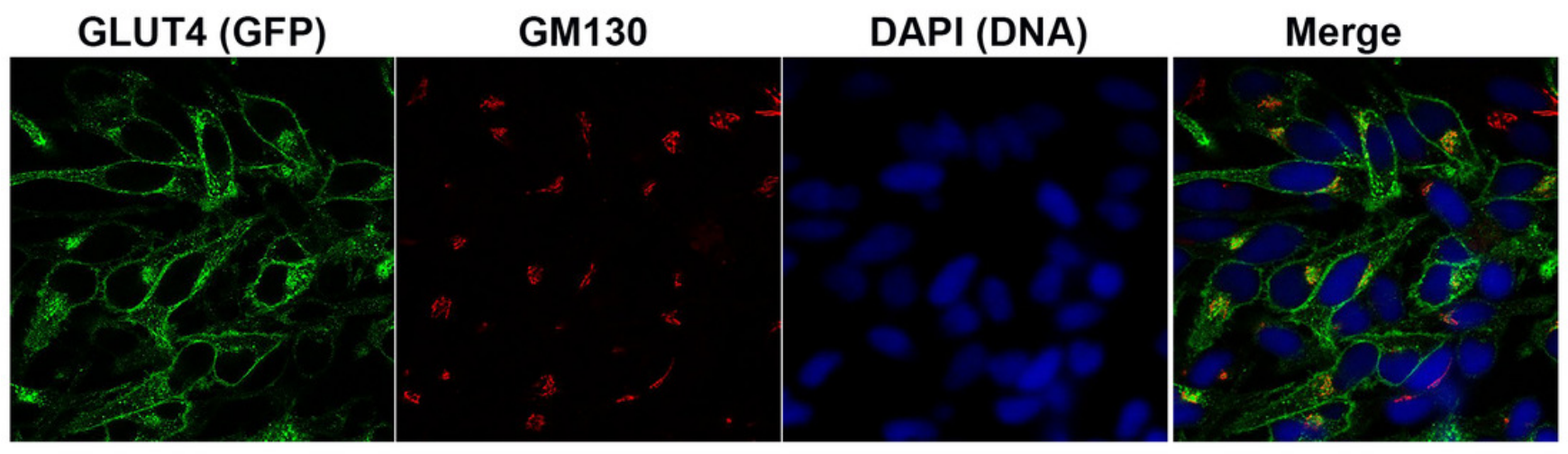

B

ERGIC53 Overlap

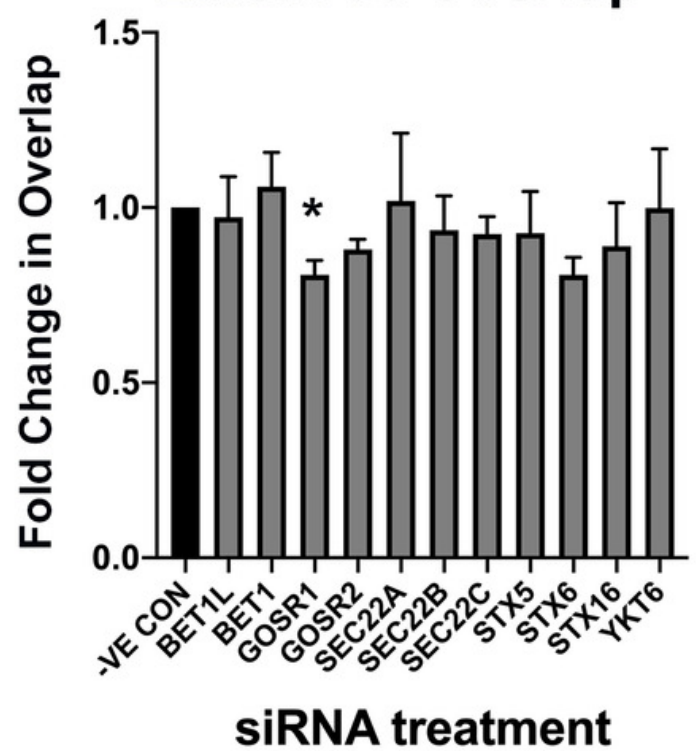

C GM130 Overlap

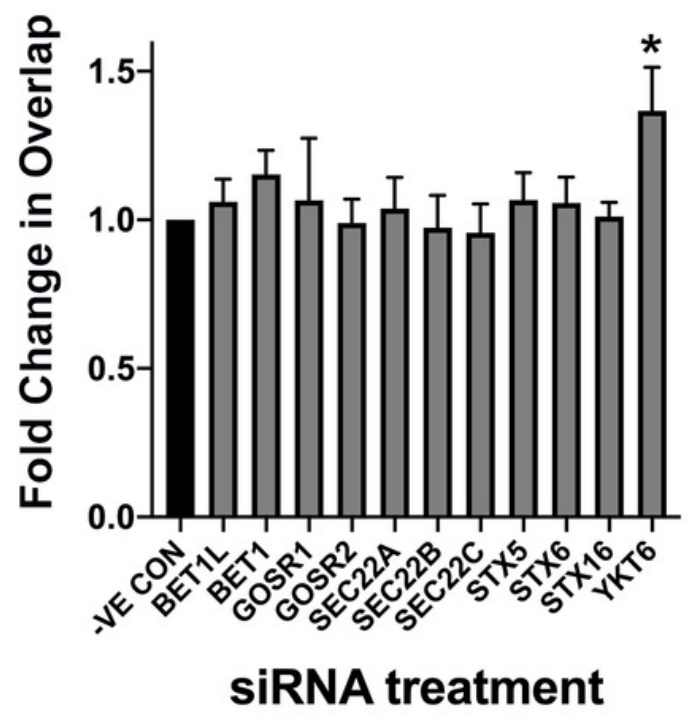

D GLUT4/DNA ratio

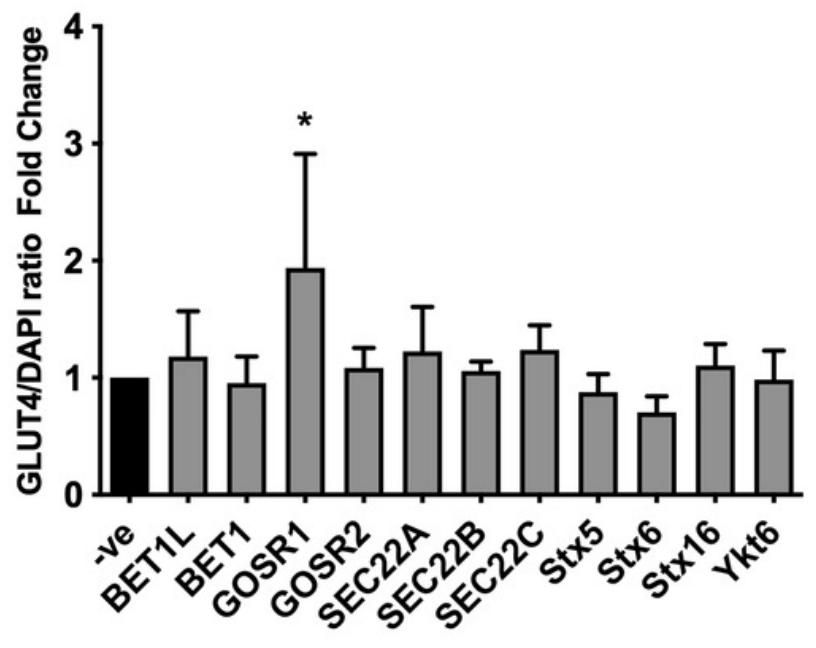

